\title{
On a metrical theorem of W. Schmidt
}

by

V. Beresnevich and V. Bernik (Minsk)

1. Introduction. Let $f_{1}, \ldots, f_{n}: \mathbb{R} \rightarrow \mathbb{R}$ be $(n+1)$-times continuously differentiable functions. Write

$$
\begin{gathered}
W\left(f_{1}^{\prime}, \ldots, f_{n}^{\prime}\right)(x)=\left(\begin{array}{ccc}
f_{1}^{\prime}(x) & \ldots & f_{n}^{\prime}(x) \\
\ldots \ldots \ldots & \ldots & \ldots \ldots \ldots \\
f_{1}^{(n)}(x) & \ldots & f_{n}^{(n)}(x)
\end{array}\right), \\
w\left(f_{1}^{\prime}, \ldots, f_{n}^{\prime}\right)(x)=\operatorname{det} W\left(f_{1}^{\prime}, \ldots, f_{n}^{\prime}\right)(x), \\
F_{n}(x)=a_{0}+a_{1} f_{1}(x)+\ldots+a_{n} f_{n}(x),
\end{gathered}
$$

where $a_{0}, a_{1}, \ldots, a_{n} \in \mathbb{Z}$. We denote by $\mathcal{F}=\mathcal{F}_{n}$ the set of all functions of the form (3). We will suppose that

$$
w\left(f_{1}^{\prime}, \ldots, f_{n}^{\prime}\right)(x) \neq 0
$$

for almost all $x$. Moreover, $\mu A$ is the Lebesgue measure of the set $A$ in $\mathbb{R}$. We are interested in the solutions of the inequalities

$$
|F(x)|<H^{-n-\varepsilon},
$$

where $H=H(F)=\max \left(\left|a_{0}\right|, \ldots,\left|a_{n}\right|\right), F \in \mathcal{F}_{n}, \varepsilon>0$. For $\varepsilon>0$ we define

(6) $\Psi=\Psi_{n}(\varepsilon)=\left\{x \in \mathbb{R}:(5)\right.$ holds for infinitely many $\left.F \in \mathcal{F}_{n}\right\}$.

In $1964 \mathrm{~W}$. Schmidt proved that $\mu \Psi_{2}=0$ (see [2]). In this article we prove the next case:

Theorem. For any $\varepsilon>0, \mu \Psi_{3}(\varepsilon)=0$.

We set

$$
\sigma(F)=\left\{x \in \mathbb{R}:|F(x)|<H^{-3-\varepsilon}\right\},
$$

where $F \in \mathcal{F}_{3}$. For any finite interval $\Delta \subset \mathbb{R}$ we put

$$
\widehat{\Delta}=\{x \in \mathbb{R}:|x-y| \leq 2 \mu \Delta \text { for any } y \in \Delta\} .
$$

We write $X \ll Y$ for $X=O(Y)$, and $X \asymp Y$ is equivalent to the simultaneous validity of $X \ll Y$ and $Y \ll X$. Moreover, $|A|$ is the number of elements in a finite set $A$. We denote by $d\left(\Delta_{1}, \Delta_{2}\right)$ the distance between the 
centers of two intervals $\Delta_{1}, \Delta_{2}$. Notice one property of $d\left(\Delta_{1}, \Delta_{2}\right)$ : suppose we have two families of intervals $\Delta_{1}(t)$ and $\Delta_{2}(t)$ which satisfy the condition

$$
\max \left(\mu \Delta_{1}(t), \mu \Delta_{2}(t)\right) \underset{t \rightarrow \infty}{=} o\left(d\left(\Delta_{1}(t), \Delta_{2}(t)\right)\right) .
$$

Then for any $x_{1}(t) \in \Delta_{1}(t)$ and $x_{2}(t) \in \Delta_{2}(t)$, we have

$$
\left|x_{1}(t)-x_{2}(t)\right| \asymp d\left(\Delta_{1}(t), \Delta_{2}(t)\right) .
$$

The proof is trivial.

Let $1 \leq m \leq n$. We denote by $C(n, m)$ the set of all $\mathbf{J}=\left(j_{1}, \ldots, j_{m}\right) \in$ $\mathbb{Z}^{m}$, where $1 \leq j_{1}<\ldots<j_{m} \leq n$, and $\left(f_{j_{1}}, \ldots, f_{j_{m}}\right)$ is denoted by $\bar{f}_{\mathbf{J}}$.

\section{Auxiliary statements}

Lemma 1. Let $M \subset \mathbb{R}$ and suppose that every point of $M$ is isolated. Then $M$ is at most countable.

Lemma 1 is well known. It is an easy exercise.

Lemma 2. Let $\varphi: \mathbb{R} \rightarrow \mathbb{R}$ be an m-times continuously differentiable function, and $N=\{x \in \mathbb{R}: \varphi(x)=0\}$. Let $\mu N>0$. Then there exists a subset $L \subset N$ such that

(a) $N \backslash L$ is at most countable,

(b) for any $i \in\{1, \ldots, m\}$ and for any $x \in L, \varphi^{(i)}(x)=0$.

Proof. It is sufficient to prove this lemma for $m=1$. We denote by $L$ the set of all limit points of $N$. Then $M=N \backslash L$ consists of all isolated points of $N$. From Lemma 1 it follows that $M$ is at most countable. Since $\varphi$ is continuous, $N$ is closed. Hence $L \subset N$. Now (b) is easy to obtain by applying the definition of limit points in terms of sequences, Lagrange's formula and the continuity of $\varphi^{\prime}$.

Lemma 3. Let $f_{i}: \mathbb{R} \rightarrow \mathbb{R}(1 \leq i \leq n)$ be $n$-times continuously differentiable functions and $w\left(f_{1}^{\prime}, \ldots, f_{n}^{\prime}\right) \neq 0$ for almost all $x \in \mathbb{R}$. Then for any $m \in\{1, \ldots, n\}$ and any $\mathbf{J} \in C(n, m)$,

$$
w\left(\bar{f}_{\mathbf{J}}^{\prime}\right) \neq 0
$$

for almost all $x \in \mathbb{R}$.

Proof. Let $m=1,1 \leq j \leq n$ and $N=\left\{x: f_{j}^{\prime}(x)=0\right\}$. Suppose $\mu N>0$. By Lemma 2 there exists $L \subset N$ such that $\mu L=\mu N>0$ and $f_{j}^{(i)}(x)=0$ for any $i=1, \ldots, n$ and for any $x \in L$. Hence for any $x \in L$ the $i$ th column in $W\left(f_{1}^{\prime}, \ldots, f_{n}^{\prime}\right)(x)$ is zero. It follows that $w\left(f_{1}^{\prime}, \ldots, f_{n}^{\prime}\right)=0$ for any $x \in L$. But $\mu L>0$. The contradiction proves the lemma for $m=1$.

Now suppose the lemma is proved for $m-1$ with $m>1$. We write $N=\left\{x: w\left(\bar{f}_{\mathbf{J}}^{\prime}\right)(x)=0\right\}$, where $\mathbf{J} \in C(n, m)$. We denote by $\bar{r}_{i}$ the $i$ th 
derivative of $\bar{f}_{\mathbf{J}}$. Suppose $\mu N>0$. According to Lemma 2 there exists $L \subset N$ such that $\mu L=\mu N>0$ and

$$
\frac{d^{k}}{d x^{k}}\left(w\left(\bar{f}_{\mathbf{J}}^{\prime}\right)\right)=0
$$

for all $x \in L$, where $1 \leq k \leq n-m$. From the inductive assumption it follows that the vectors $\bar{r}_{1}(x), \ldots, \bar{r}_{m-1}(x)$ are linearly independent for almost all $x \in \mathbb{R}$. Hence we can assume that they are linearly independent for all $x \in L$. Applying (12) with $k=1, \ldots, n-m$ we find that $\bar{r}_{i}(x)$ depends linearly on $\bar{r}_{1}(x), \ldots, \bar{r}_{m-1}(x)$ for all $x \in L, 1 \leq i \leq n$. Hence the columns of $W\left(f_{1}^{\prime}, \ldots, f_{n}^{\prime}\right)$ with indices $j_{1}, \ldots, j_{m}$ are linearly dependent for all $x \in L$. This contradiction finishes the proof.

Define

$$
S=\bigcup_{m=1}^{n} \bigcup_{\mathbf{J} \in C(n, m)}\left\{x \in \mathbb{R}: w\left(\bar{f}_{\mathbf{J}}^{\prime}\right)(x)=0\right\} .
$$

Since $S$ is closed, $\mathbb{R} \backslash S$ has the form $\bigcup_{k=1}^{\infty}\left[a_{k}, b_{k}\right]$. From Lemma 3 it follows that $\mu S=0$. Then

$$
\mu \Psi \leq \sum_{k=1}^{\infty} \mu\left(\Psi \cap\left[a_{k}, b_{k}\right]\right) .
$$

In order to prove our theorem it is sufficient to show that if $I=[a, b]$ and $I \cap S=\emptyset$ then $\mu(\Psi \cap I)=0$. Later on, to simplify the writing, we let $I$ be a fixed closed interval in $\mathbb{R} \backslash S$. We redefine $\sigma(F)$ and $\Psi$ to be the intersection of $I$ with the former sets $\sigma(F)$ and $\Psi$. Since $w\left(\bar{f}_{\mathbf{J}}^{\prime}\right)$ is continuous and not zero over $I$, for all $\mathbf{J} \in C(n, m)$ with $1 \leq m \leq n$ and for all $x \in I$ we have

$$
\left|w\left(\bar{f}_{\mathbf{J}}^{\prime}\right)(x)\right| \geq d>0
$$

where $d$ is a positive constant depending on the functions $f_{1}, \ldots, f_{n}$ and the interval $I$ only.

Lemma 4. Let $\delta, \nu>0$. Let $\varphi$ be an n-times continuously differentiable function on $(a, b)$ satisfying $\left|\varphi^{(n)}(x)\right| \geq \delta$ for all $x \in(a, b)$. Then $\mu(\{x \in$ $(a, b):|\varphi(x)|<\nu\}) \leq c(n)(\nu / \delta)^{(1 / n)}$.

This is proved in [1].

LEMMA 5. Set $\alpha_{m}=\max \left\{1, \sup \left\{\left|f_{j}^{(i)}(x)\right|: x \in I\right\}: 0 \leq i \leq m, 1 \leq j\right.$ $\leq n\}$ and $C_{1}=d \alpha_{n}^{-n} /(n+1)$ !, where $f_{i} \in C^{(n)}(\mathbb{R})(1 \leq i \leq n)$. Then for all $x \in \sigma(F)$ and $H \geq H_{0}$ we have

$$
\max _{1 \leq i \leq n}\left(\left|F^{(i)}(x)\right|\right) \geq C_{1} H
$$

where $\sigma(F)$ is defined in $(7), F \in \mathcal{F}_{n}$ and $H=H(F)$. 
Pr o of. We may write the following system of linear equations:

$$
\left\{\begin{array}{l}
a_{0}+a_{1} f_{1}(x)+\ldots+a_{n} f_{n}(x)=F(x), \\
a_{1} f_{1}^{\prime}(x)+\ldots+a_{n} f_{n}^{\prime}(x)=F^{\prime}(x), \\
\ldots \ldots \ldots \ldots \ldots \ldots \ldots \ldots \ldots \ldots \ldots \ldots \ldots \ldots \ldots \\
a_{1} f_{1}^{(n)}(x)+\ldots \ldots+a_{n} f_{n}^{(n)}(x)=F^{(n)}(x) .
\end{array}\right.
$$

The modulus of the determinant of (15) is not less than $d$. Using Cramer's rule we have, for $i=0, \ldots, n$,

$$
\left|a_{i}\right| \leq \frac{1}{d} \alpha_{n}^{n}(n+1) ! \max \left\{\left|F^{(j)}(x)\right|: 0 \leq j \leq n\right\},
$$

whence the lemma readily follows.

Lemma 6. Let $f_{i}(1 \leq i \leq n)$ be $(n+1)$-times continuously differentiable functions. Suppose $C_{2}=C_{1} /\left(2 n \alpha_{n+1}\right), \mu I \leq C_{2}$ and $\left|F^{(i)}(\kappa)\right| \geq C_{1} H$, where $\kappa \in I$ and $1 \leq i \leq n$. Then $\left|F^{(i)}(x)\right| \geq C_{1} H / 2$ for all $x \in I$.

Proof. Assume $x \in I$. By Lagrange's formula, $F^{(i)}(x)=F^{(i)}(\kappa)+$ $F^{(i+1)}\left(\kappa_{1}\right)(x-\kappa)$. Furthermore, $\left|F^{(i+1)}(\kappa)(x-\kappa)\right| \leq n \alpha_{n+1} C_{2} H=C_{1} H / 2$. Thus $\left|F^{(i)}(x)\right| \geq\left|F^{(i)}(\kappa)\right|-\left|F^{(i+1)}\left(\kappa_{1}\right)(x-\kappa)\right| \geq C_{1} H-C_{1} H / 2$, and the lemma is proved.

Since $I$ is a finite union of intervals of length $\leq C_{2}$, we may suppose without loss of generality that $\mu I \leq C_{2}$.

3. Preliminary remarks. From now on, $n=3$.

Remark 1. Suppose we have a finite set of conditions according to which $\mathcal{F}$ is divided into subclasses: $\mathcal{F}=\bigcup_{i=1}^{N} \mathcal{F}^{i}$. Let a division of $\sigma(F)=$ $\bigcup_{j=1}^{M} \sigma^{j}(F)$ into a finite number of intervals be defined for every $F \in \mathcal{F}$, where $M$ is an absolute constant. Define

$$
\Psi_{i, j}=\bigcap_{k=1}^{\infty} \bigcup_{F \in \mathcal{F}^{i}, H(F) \geq k} \sigma^{j}(F) .
$$

Then

$$
\Psi=\bigcap_{k=1}^{\infty} \bigcup_{F \in \mathcal{F}, H(F) \geq k} \sigma(F) \subset \bigcup_{i=1}^{N} \bigcup_{j=1}^{M} \Psi_{i, j} .
$$

Hence if we prove that $\mu \Psi_{i, j}=0$ for all $1 \leq i \leq N, 1 \leq j \leq M$, we obtain $\mu \Psi=0$. In the sequel to simplify the writing we shall impose some conditions, additional indices being omitted.

Remark 2. From Lemmas 5 and 6 it follows that there exists $k \in$ $\{1,2,3\}$ such that $\left|F^{(k)}(x)\right| \geq C_{1} H / 2$. We obtain a covering of $I$ by at most six subintervals such that $F^{(i)}(x)$ is monotone on each subinterval for 
$0 \leq i \leq k-1$, where $F^{(0)} \equiv F$. Therefore by Remark 1 we can assume that $\sigma(F)$ is such an interval.

Remark 3. We define

$$
\mathcal{F}(t)=\left\{F \in \mathcal{F}: 2^{t} \leq H(F) \leq 2^{t+1}\right\} .
$$

The number of functions in $\mathcal{F}(t)$ is $\ll 2^{4 t}$. Suppose we have $\mu \sigma(F) \ll H^{-4-\xi}$ for some $\xi>0$. Then

$$
\sum_{F \in \mathcal{F}(t)} \mu \sigma(F) \ll 2^{-\xi t}
$$

The convergence of $\sum 2^{-\xi t}$ and the Borel-Cantelli lemma now show that the set of $x$ belonging to infinitely many sets of $\sigma(F)$ has measure zero.

Remark 4. Lemmas 4-6 give the estimate

$$
\mu \sigma(F) \ll H^{-(4+\varepsilon) / 3} .
$$

If $\varepsilon>8$ then from (19) we get $\mu \sigma(F) \ll H^{-4-\xi}$, where $\xi=(\varepsilon-8) / 3$, and Remark 3 yields the assertion of the Theorem. Therefore below we consider $\varepsilon \leq 8$.

Remark 5. If $\left|F^{\prime}(x)\right| \geq H^{1-\varepsilon / 2}$ for $x \in \sigma(F)$ then we get the estimate $\mu \sigma(F) \ll H^{-4-\varepsilon / 2}$. If $\left|F^{\prime}(x)\right|<H^{-9}$ for $x \in \sigma(F)$ then $\mu \sigma(F) \ll H^{-5}$. If $\left|F^{\prime \prime}(x)\right|<H^{-4}$ then $\mu \sigma(F) \ll H^{-5}$. These estimates readily follow from Lemma 4 with $\varphi$ equal to $F^{\prime}$ and $F^{\prime \prime}$ respectively. In each of these cases, Remark 3 yields the assertion of the Theorem. Therefore further we may suppose that

$$
\begin{gathered}
\left|F^{\prime}(x)\right|<H^{1-\varepsilon / 2}, \\
\left|F^{\prime}(x)\right| \geq H^{-9}, \quad\left|F^{\prime \prime}(x)\right| \geq H^{-4}
\end{gathered}
$$

for $x \in \sigma(F)$.

Choose a positive parameter

$$
\delta=\min \left(\frac{\varepsilon}{20}, \frac{\varepsilon^{2}}{4(5+\varepsilon)}, \frac{\varepsilon^{2}}{16(4+\varepsilon)}\right) .
$$

The conditions

$$
\begin{aligned}
H^{(l-1) \delta} & \leq\left|F^{\prime}(x)\right|<H^{l \delta}, \\
H^{(k-1) \delta} & \leq\left|F^{\prime \prime}(x)\right|<H^{k \delta},
\end{aligned}
$$

where $k, l \in \mathbb{Z}$, define a subdivision of $\sigma(F)$. If $(l-1) \delta>1$ or $(k-1) \delta>1$ then the corresponding element of the subdivision is empty when $H \geq H_{0}$. From (21) we have $l \delta \geq-9, k \delta \geq-4$. Hence the number of different integers $(k, l)$ is finite. We can thus suppose that $\sigma(F)$ is an interval and conditions (23) and (24) hold for all $x \in \sigma(F)$, where $k$ and $l$ are fixed. 


\section{Proof of the Theorem. The case of large first derivative}

Proposition 1. Let $(l-1) \delta \geq-1-\varepsilon / 4$ and suppose condition (23) holds for $x \in \sigma(F)$. Then the measure of those $x \in I$ which belong to infinitely many $\sigma(F)$ is at most $\mu \Psi(\varepsilon+\varepsilon / 8)$.

P r o of. The considered functions $F$ are divided into the subclasses $\mathcal{F}(t)$ defined in (17). Suppose $\eta=3+3 \varepsilon / 4+(l-1) \delta$. Using Lemma 4 and (23) we get

$$
\mu \sigma(F) \ll H^{-3-\varepsilon-(l-1) \delta} .
$$

We define

$$
[\Delta]_{t}=\{F \in \mathcal{F}(t): \sigma(F) \cap \Delta \neq \emptyset\}
$$

for any interval $\Delta \subset I$. For every fixed $t$ we divide $I$ into subintervals $I_{s}^{t}$ of length $c n^{-\eta t}$ each, where $c=c(t) \in[1,2]$.

The number of different $I_{s}^{t}$ is $\ll 2^{\eta t}$. Now define

$$
\mathcal{F}^{\prime}(t)=\bigcup_{s}\left[I_{s}^{t}\right]
$$

where the union is taken over those $I_{s}^{t}$ for which $\left|\left[I_{s}^{t}\right]_{t}\right| \leq 2^{(\varepsilon / 4-\delta) t}$. We consider

$$
\mathcal{F}^{\prime \prime}(t)=\mathcal{F}(t) \backslash \mathcal{F}^{\prime}(t), \quad \mathcal{F}^{\prime}=\bigcup_{t} \mathcal{F}^{\prime}(t), \quad \mathcal{F}^{\prime \prime}=\bigcup_{t} \mathcal{F}^{\prime \prime}(t) .
$$

Counting the number of functions in $\mathcal{F}^{\prime}(t)$ and using (25) we get

$$
\begin{aligned}
\sum_{t \geq 0} \sum_{F \in \mathcal{F}^{\prime}(t)} \mu \sigma(F) & \ll \sum_{t \geq 0} 2^{\eta t} 2^{(\varepsilon / 4-\delta) t} 2^{(-3-\varepsilon-(l-1) \delta) t} \\
& =\sum_{t \geq 0} 2^{-\delta t}<\infty
\end{aligned}
$$

Thus, from the Borel-Cantelli lemma it follows that the set of those $x \in I$ which belong to infinitely many $\sigma(F)$ for $F \in \mathcal{F}^{\prime}$ has measure zero.

Now consider $x_{0} \in I$ belonging to infinitely many $\sigma(F)$ for $F \in \mathcal{F}^{\prime \prime}$. The choice of $\eta$ and the estimate (25) show that $\sigma(F) \subset \widehat{I}_{s}^{t}$ if $t \geq t_{0}$ and $F \in\left[I_{s}^{t}\right]_{t}$. Thus $x_{0}$ belongs to $\widehat{I}_{s}^{t}$ for infinitely many $t$ with $\left|\left[I_{s}^{t}\right]_{t}\right|>2^{(\varepsilon / 4-\delta) t}$. Consider a fixed such interval $I_{s}^{t}$. Let $F \in\left[I_{s}^{t}\right]_{t}$ and $\kappa \in \sigma(F) \cap I_{s}^{t}$. By Taylor's formula we have

$$
F(x)=F(\kappa)+F^{\prime}(\kappa)(x-\kappa)+\frac{1}{2} F^{\prime \prime}\left(\kappa_{1}\right)(x-\kappa)^{2},
$$

where $\kappa_{1}$ lies between $x$ and $\kappa$. From (5), (23) and the estimate $|x-\kappa| \ll H^{-\eta}$ we get

$$
|F(x)| \ll H^{-3-\varepsilon}+H^{l \delta-\eta}+H^{1-2 \eta} .
$$


The choice of $\delta$ and $\eta$ and the assumption of Proposition 1 imply that the first and third terms on the right hand side (30) are less than the second term. Now using the value of $\eta$, and (30), we obtain

$$
|F(x)| \ll H^{-3-3 \varepsilon / 4+\delta}
$$

for all $x \in \widehat{I}_{s}^{t}$. Analogously we have

$$
\left|F^{\prime}(x)\right| \ll H^{l \delta}
$$

for all $x \in \widehat{I}_{s}^{t}$.

Both $a_{2}$ and $a_{3}$ range in the interval $\left[-2^{t+1}, 2^{t+1}\right]$. We divide it into intervals $\Delta_{j}$ with length $2^{t(1-\varepsilon / 8+\delta / 2)+2}$. Thus we obtain at most $2^{t(\varepsilon / 4-\delta)}$ pairs of intervals $\left(\Delta_{j_{1}}, \Delta_{j_{2}}\right)$. Since by assumption we have $\left|\left[I_{s}^{t}\right]_{t}\right|>2^{t(\varepsilon / 4-\delta)}$, there exist $F_{1}, F_{2} \in\left[I_{s}^{t}\right]_{t}$ whose coefficients $a_{2}$ and $a_{3}$ belong to one pair of intervals $\left(\Delta_{j_{1}}, \Delta_{j_{2}}\right)$. Consider $R(x)=F_{1}(x)-F_{2}(x)$. We obtain

$$
\left|a_{i}(R)\right| \leq 2^{t(1-\varepsilon / 8+\delta / 2)+2}
$$

for $i=2,3$. From (31) and (32) for $F_{1}$ and $F_{2}$ it follows that

$$
\begin{gathered}
|R(x)| \ll 2^{t(-3-3 \varepsilon / 4+\delta)}, \\
\left|R^{\prime}(x)\right| \ll 2^{l \delta t}
\end{gathered}
$$

for all $x \in \widehat{I}_{s}^{t}$. From (20) we get $l \delta \leq 1-\varepsilon / 2+\delta<1-\varepsilon / 8+\delta / 2$. Therefore from (13), (33) and (35) we have $\left|a_{1}(R)\right| \ll 2^{t(1-\varepsilon / 8+\delta / 2)}$. From this and (34) we obtain $\left|a_{0}(R)\right| \ll 2^{t(1-\varepsilon / 8+\delta / 2)}$. Thus we conclude that

$$
H(R) \ll 2^{t(1-\varepsilon / 8+\delta / 2)} .
$$

The relation

$$
|R(x)| \ll H(R)^{-(3+3 \varepsilon / 4-\delta) /(1-\varepsilon / 8+\delta / 2)}
$$

follows from (34) and (36) for all $x \in \widehat{I}_{s}^{t}$. We have

$$
\frac{3+3 \varepsilon / 4-\delta}{1-\varepsilon / 8+\delta / 2}-(3+\varepsilon)>3+3 \varepsilon / 4-\delta-(1-\varepsilon / 8+\delta / 2)(3+\varepsilon) \geq \varepsilon / 8 .
$$

Therefore

$$
\left|R\left(x_{0}\right)\right|<H(R)^{-3-\varepsilon-\varepsilon / 8},
$$

where $H(R) \geq H_{0}$ and $H_{0}$ is sufficiently large.

Remark 6. Applying Lemma 2 it is easy to show that for every fixed $R \in \mathcal{F}_{n}$ the measure of $E_{n}(R)=\{x: R(x)=0\}$ is zero. Then the union $E_{n}$ of all $E_{n}(R)$ with $R \in \mathcal{F}_{n}$ also has measure zero. If the number of different $R(x)$ in (38) is finite then $x_{0}$ is a solution of some equivalent $R(x)=0$, where $R$ has the form (3).

The inequality (38) and the previous discussion prove Proposition 1. 


\section{The case of small second derivative}

Proposition 2. Let $(l-1) \delta<-1-\varepsilon / 4,(k-1) \delta \leq 1-\varepsilon / 2$ and suppose that conditions (23) and (24) are valid for $x \in \sigma(F)$. Then the measure of the set of $x \in I$ belonging to infinitely many $\sigma(F)$ is at most $\mu \Psi\left(\varepsilon+\varepsilon^{2} / 16\right)$.

Pro of. From (23), (24) and Lemmas 5 and 6 it follows that

$$
\left|F^{\prime \prime \prime}(x)\right| \geq C_{1} H / 2
$$

for all $x \in I$. By Lemma 4, from (5), (23), (24) and (39) we get six estimates of the measure of $\sigma(F)$. Choosing the optimal estimate we obtain

$$
\mu \sigma(F) \ll H^{-\nu},
$$

where

$$
\begin{aligned}
\nu=\max \left(3+\varepsilon+(l-1) \delta, \frac{3+\varepsilon+(k-1) \delta}{2}, \frac{4+\varepsilon}{3},\right. \\
\left.\quad-l \delta+(k-1) \delta, \frac{-l \delta+1}{2},-k \delta+1\right) .
\end{aligned}
$$

Suppose $\eta=\nu-\varepsilon / 8$. We divide all the functions $F \in \mathcal{F}_{3}$ under consideration into the subclasses $\mathcal{F}(t)$ defined in (17). For every fixed integer $t$ we divide $I$ into subintervals $I_{s}^{t}$ of length $c 2^{-\eta t}$ each, where $c=c(t) \in[1,2]$. The number of different $I_{s}^{t}$ is $\ll 2^{\eta t}$. The classes $\mathcal{F}^{\prime}(t)$ and $\mathcal{F}^{\prime \prime}(t)$ are defined in the same way as in (27) and (28), with the union in (27) taken over those $I_{s}^{t}$ for which $\left|\left[I_{s}^{t}\right]_{t}\right| \leq 2^{t(\varepsilon / 8-\delta)}$. The classes $\mathcal{F}^{\prime}$ and $\mathcal{F}^{\prime \prime}$ are defined as above. Counting the number of functions in $\mathcal{F}^{\prime}(t)$ and using (40) we get

$$
\sum_{t \geq 0} \sum_{F \in \mathcal{F}^{\prime}(t)} \mu \sigma(F) \ll \sum_{t \geq 0} 2^{\eta t} 2^{(\varepsilon / 8-\delta) t} 2^{-\nu t}=\sum_{t \geq 0} 2^{-\delta t}<\infty .
$$

Thus the Borel-Cantelli lemma shows that the set of those $x \in I$ which belong to infinitely many $\sigma(F)$ for $F \in \mathcal{F}^{\prime}$ has zero measure.

Now consider $x_{0} \in I$ belonging to infinitely many $\sigma(F)$ for $F \in \mathcal{F}^{\prime \prime}$. The choice of $\eta$ and the estimate (40) give $\sigma(F) \subset \widehat{I}_{s}^{t}$ if $t \geq t_{0}$ and $F \in\left[I_{s}^{t}\right]_{t}$. Thus $x_{0}$ belongs to $\widehat{I}_{s}^{t}$ for infinitely many $t$ and $\left|\left[I_{s}^{t}\right]_{t}\right|>2^{(\varepsilon / 4-\delta) t}$. Consider a fixed such interval $I_{s}^{t}$. Let $F \in\left[I_{s}^{t}\right]_{t}$ and $\kappa \in \sigma(F) \cap I_{s}^{t}$. From (24) and Taylor's formula we obtain

$$
\begin{aligned}
\left|F^{\prime \prime}(x)\right| & =\left|F^{\prime \prime}(\kappa)+F^{\prime \prime \prime}\left(\kappa_{1}\right)(x-\kappa)\right| \leq\left|F^{\prime \prime}(\kappa)\right|+\left|F^{\prime \prime \prime}\left(\kappa_{1}\right)(x-\kappa)\right| \\
& \ll H^{k \delta}+H^{1-\eta} \leq H^{k \delta}+H^{k \delta+\varepsilon / 8} \leq 2 \cdot H^{k \delta+\varepsilon / 8},
\end{aligned}
$$

where $\kappa_{1}$ lies between $x$ and $\kappa$. Analogously we get estimates for $F(x)$ and $F^{\prime}(x)$ using (23), (24) and Taylor's formula. Thus

$$
\begin{gathered}
|F(x)| \ll H^{-3-\varepsilon+3 \varepsilon / 8+\delta}, \\
\left|F^{\prime}(x)\right| \ll H^{l \delta+2 \varepsilon / 8+\delta},
\end{gathered}
$$




$$
\left|F^{\prime \prime}(x)\right| \ll H^{k \delta+\varepsilon / 8}
$$

for all $x \in \widehat{I}_{s}^{t}$. The coefficient $a_{3}$ ranges over the interval $\left[-2^{t+1}, 2^{t+1}\right]$. We divide it into intervals $\Delta_{j}$ of length $2^{t(1-\varepsilon / 8+\delta)+2}$. There are at most $2^{t(\varepsilon / 8-\delta)}$ intervals $\Delta_{j}$. Since by assumption we have $\left|\left[I_{s}^{t}\right]_{t}\right|>2^{t(\varepsilon / 8-\delta)}$ there exist $F_{1}, F_{2} \in\left[I_{s}^{t}\right]_{t}$ whose coefficients $a_{3}$ belong to one $\Delta_{j}$. Consider $R(x)=$ $F_{1}(x)-F_{2}(x)$. Then

$$
\left|a_{3}(R)\right| \leq 2^{t(1-\varepsilon / 8+\delta)+2} .
$$

It is clear that conditions (41)-(43) apply to $R(x)$ if we substitute $2^{t}$ for $H$. It is not difficult to verify that $l \delta+2 \varepsilon / 8+\delta \leq 1-\varepsilon / 8+\delta$ and $k \delta+\varepsilon / 8 \leq$ $1-\varepsilon / 8+\delta$. From conditions (42) and (43) for $F_{1}$ and $F_{2}$ it follows that

$$
\left|R^{\prime}(x)\right| \ll 2^{t(1-\varepsilon / 8+\delta)}, \quad\left|R^{\prime \prime}(x)\right| \ll 2^{t(1-\varepsilon / 8+\delta)} .
$$

By (44) and (45),

$$
\begin{aligned}
\mid a_{1}(R) f_{1}^{\prime}(x)+a_{2}(R) f_{2}^{\prime}(x) & \ll 2^{t(1-\varepsilon / 8+\delta)}, \\
\left|a_{1}(R) f_{1}^{\prime \prime}(x)+a_{2}(R) f_{2}^{\prime \prime}(x)\right| & \ll 2^{t(1-\varepsilon / 8+\delta)} .
\end{aligned}
$$

From (46) we obtain $\left|a_{i}(R)\right| \ll 2^{t(1-\varepsilon / 8+\delta)}(i=1,2)$ because $\left|w\left(f_{1}^{\prime}, f_{2}^{\prime}\right)\right| \geq$ $d>0$ according to (13). From (41) for $F_{1}$ and $F_{2}$ it follows that

$$
|R(x)| \ll 2^{t(-3-\varepsilon+3 \varepsilon / 8+\delta)}
$$

and from (47) we find $\left|a_{0}(R)\right| \ll 2^{t(1-\varepsilon / 8+\delta)}$. Hence

$$
H(R) \ll 2^{t(1-\varepsilon / 8+\delta)} .
$$

Observe that

$\frac{3-\varepsilon-3 \varepsilon / 8-\delta}{1-\varepsilon / 8+\delta}-(3-\varepsilon)>3+\varepsilon-3 \varepsilon / 8-\delta-(1-\varepsilon / 8+\delta)(3+\varepsilon) \geq \varepsilon^{2} / 16$.

Thus from (47) and (48) we obtain

$$
\left|R\left(x_{0}\right)\right|<H(R)^{-3-\varepsilon-\varepsilon^{2} / 16}
$$

with $H(R) \geq H_{0}$, where $H_{0}$ is sufficiently large. The last inequality together with Remark 6 finishes the proof of Proposition 2.

6. The last case. Let $\gamma>0$. Set

$$
\mathcal{G}=\left\{F=a_{0}+a_{1} f_{1}+a_{2} f_{2}:\left(a_{0}, a_{1}, a_{2}\right) \in \mathbb{Z}^{3} \backslash\{0\}\right\} .
$$

For $F \in \mathcal{G}$ consider the system

$$
|F(x)|<H^{-1-\gamma}, \quad\left|F^{\prime}(x)\right|<H^{-\gamma / 2},
$$

where $H=H(F)=\max \left(\left|a_{0}\right|,\left|a_{1}\right|,\left|a_{2}\right|\right)$. The set of its solutions is denoted by $\sigma^{*}(F)$. Define

(50) $\Omega(\gamma)=\{x \in I:(49)$ is valid for infinitely many $F \in \mathcal{G}\}$. 
Now we return to our problem. By Remark 1 we can assume that any $F \in \mathcal{F}_{3}$ has $a_{3} \geq\left|a_{i}\right|(1 \leq i \leq 3)$.

Proposition 3. Let $(l-1) \delta<-1-\varepsilon / 4,(k-1) \delta>1-\varepsilon / 2$ and suppose that conditions (23) and (24) are valid throughout $\sigma(F)$. Moreover, let $a_{3} \geq$ $\left|a_{i}\right|(1 \leq i \leq 3)$ for $F \in \mathcal{F}_{3}$. Then the measure of the set of $x \in I$ belonging to infinitely many $\sigma(F)$ is at most $\mu \Omega(\varepsilon / 5)$.

Proof. We have $|F(x)| \geq H^{1-\varepsilon / 2}$. By Lemma 4 we get $\mu \sigma(F) \ll$ $H^{-2-\varepsilon / 4}$. Define $\eta=1+\varepsilon / 8$. Divide the collection of $F \in \mathcal{F}_{3}$ under consideration into the subclasses $\mathcal{F}(t)=\left\{F \in \mathcal{F}_{3}: a_{3}(F)=t\right\}$. It is clear that $H(F) \asymp t$ for $F \in \mathcal{F}(t)$. Fix $t$ and divide $I$ into subintervals $I_{s}^{t}$ of length $c t^{-\eta}$ each, where $c=c(t) \in[1,2]$. The number of different $I_{s}^{t}$ is $\ll t^{\eta}$. The classes $\mathcal{F}^{\prime}(t)$ and $\mathcal{F}^{\prime \prime}(t)$ are defined as in (27) and (28), with the union in (27) taken over those $I_{s}^{t}$ for which $\left|\left[I_{s}^{t}\right]_{t}\right| \leq 1$. The classes $\mathcal{F}^{\prime}$ and $\mathcal{F}^{\prime \prime}$ are defined as above. Counting the number of functions in $\mathcal{F}^{\prime}(t)$ and estimating the measure of $\sigma(F)$ we get

$$
\sum_{t \geq 1} \sum_{F \in \mathcal{F}^{\prime}(t)} \mu \sigma(F) \ll \sum_{t \geq 1} t^{\eta} t^{-2-\varepsilon / 4}=\sum_{t \geq 1} t^{-1-\varepsilon / 8}<\infty .
$$

Thus, the Borel-Cantelli lemma shows that the set of those $x \in I$ which belong to infinitely many $\sigma(F)$ for $F \in \mathcal{F}^{\prime}$ has measure zero.

Now consider $x_{0} \in I$ belonging to infinitely many $\sigma(F)$ with $F \in \mathcal{F}^{\prime \prime}$. The choice of $\eta$ implies that $\sigma(F) \subset \widehat{I}_{s}^{t}$ if $t \geq t_{0}$, where $F \in\left[I_{s}^{t}\right]_{t}$. Thus $x_{0}$ belongs to $\widehat{I}_{s}^{t}$ for infinitely many $t$ with $\left|\left[I_{s}^{t}\right]_{t}\right| \geq 2$. Consider a fixed such interval $I_{s}^{t}$. Let $F \in\left[I_{s}^{t}\right]_{t}$ and $\kappa \in \sigma(F) \cap I_{s}^{t}$. By Taylor's formula we have $F^{\prime}(x)=F^{\prime}(\kappa)+F^{\prime \prime}\left(\kappa_{1}\right)(x-\kappa)$. Hence

$$
\left|F^{\prime}(x)\right| \ll H^{-\varepsilon / 8} \text {. }
$$

Analogously we find

$$
|F(x)| \ll H^{-1-\varepsilon / 4}
$$

for all $x \in \widehat{I}_{s}^{t}$. There exist different $F_{1}, F_{2} \in\left[I_{s}^{t}\right]_{t}$. Consider $R=F_{1}-F_{2}$. Then $R \in \mathcal{G}$ and $H(R) \ll t$. From (51) and (52) it follows that

$$
|R(x)|<H(R)^{-1-\varepsilon / 5}, \quad\left|R^{\prime}(x)\right|<H(R)^{-\varepsilon / 10},
$$

whenever $H(R) \geq H_{0}$. Thus Proposition 3 is proved.

Proposition 4. For any $\gamma>0, \mu \Omega(\gamma)=0$.

Proof. We shall consider only those $F \in \mathcal{G}$ for which $\sigma^{*}(F) \neq \emptyset$. As in the proof of Lemmas 5 and 6 , for all $x \in I$ we obtain

$$
\left|F^{\prime \prime}(x)\right| \geq C_{3} H
$$

where $F \in \mathcal{G}, H=H(F)$ and $C_{3}$ is a fixed positive constant. Moreover, from the condition $\left|a_{i}\right|=o(H)$, where $1 \leq i \leq 2$, we would get a contradiction. 
Therefore we assume that

$$
\min \left(\left|a_{1}\right|,\left|a_{2}\right|\right) \geq C_{4} H
$$

where $H=H(F)$ with $F=a_{0}+a_{1} f_{1}+a_{2} f_{2}$. Now we deal with the inequalities

$$
\begin{aligned}
& |F(x)|<H^{-1-\gamma}, \\
& \left|F^{\prime}(x)\right|<H^{-\gamma / 2}
\end{aligned}
$$

with $F \in \mathcal{G}$. Using Lemma 4 and condition (53) we find that for (55) the measure of the solution set is $\ll H^{-1-\gamma / 2}$, and similarly for (56). Thus

$$
\mu \sigma(F) \ll H^{-1-\gamma / 2},
$$

where $\sigma^{\prime}(F)$ denotes the union of the solution sets for (55) and (56). Since $\sigma^{*}(F) \neq \emptyset$ we can assume that $\sigma^{\prime}(F)$ is an interval. Moreover, $\sigma^{*}(F) \subset$ $\sigma^{\prime}(F)$.

Condition (53) implies the monotonicity of $F^{\prime}(x)$ in $I=[a, b]$. Consider those $F \in \mathcal{G}$ which have a nonvanishing derivative on all $I$. Either $a$ or $b$ necessarily belongs to $\sigma^{\prime}(F)$ because $F^{\prime}$ is monotonic. Thus there exist $C_{5}>0$ and $H_{0}$ such that for any $H \geq H_{0}$ and for all $F \in \mathcal{G}$ with $H(F) \geq H$ we have

$$
\sigma(F) \subset\left[a, a+C_{5} H^{-1-\gamma / 2}\right] \cup\left[b-C_{5} H^{-1-\gamma / 2}, b\right] .
$$

Hence $\mu \Omega(\gamma) \ll H^{-1-\gamma / 2}$ and $\mu \Omega(\gamma)=0$.

The remaining case is when $F^{\prime}(x)$ has a root $\kappa=\kappa(F) \in I$ for $F \in \mathcal{G}$.

We use the following notations: $\mathbf{A}=\left(a_{0}, a_{1}, a_{2}\right)$ is a vector; $F_{\mathbf{A}}=a_{0}+$ $a_{1} f_{1}+a_{2} f_{2} ; \mathbf{F}(x)=\left(1, f_{1}(x), f_{2}(x)\right) \in \mathbb{R}^{3} ;(\mathbf{A}, \mathbf{B})$ is the scalar product of the vectors $\mathbf{A}$ and $\mathbf{B} ; \mathbf{A} \times \mathbf{B}$ is their vector product. Set $g(x)=f_{2}^{\prime}(x) / f_{1}^{\prime}(x)$. Then

$$
g^{\prime}(x)=\frac{f_{1}^{\prime \prime}(x) f_{2}^{\prime}(x)-f_{2}^{\prime \prime}(x) f_{1}^{\prime}(x)}{\left(f_{1}^{\prime}(x)\right)^{2}} .
$$

From (13) and (58) it follows that $g^{\prime}(x) \neq 0$ for all $x \in I$. Hence $g^{\prime}(x) \asymp 1$. Let $F_{\mathbf{A}}, F_{\mathbf{B}} \in \mathcal{G}$, and let $\kappa_{\mathbf{A}}$ and $\kappa_{\mathbf{B}}$ be the roots of $F_{\mathbf{A}}^{\prime}$ and $F_{\mathbf{B}}^{\prime}$ respectively. Obviously $g\left(\kappa_{\mathbf{A}}\right)=a_{1} / a_{2}$ and $g\left(\kappa_{\mathbf{B}}\right)=b_{1} / b_{2}$.

We have

$$
\left|a_{1} / a_{2}-b_{1} / b_{2}\right|=\left|g\left(\kappa_{\mathbf{A}}\right)-g\left(\kappa_{\mathbf{B}}\right)\right|=\left|g^{\prime}(\tau)\left(\kappa_{\mathbf{A}}-\kappa_{\mathbf{B}}\right)\right| \asymp\left|\left(\kappa_{\mathbf{A}}-\kappa_{\mathbf{B}}\right)\right|,
$$

where $\tau$ lies between $\kappa_{\mathbf{A}}$ and $\kappa_{\mathbf{B}}$. We obtain

$$
\left|a_{1} / a_{2}-b_{1} / b_{2}\right| \asymp\left|\kappa_{\mathbf{A}}-\kappa_{\mathbf{B}}\right| .
$$

We divide the considered $F \in \mathcal{G}$ into the classes

$$
G(t)=\left\{F \in \mathcal{G}: 2^{t} \leq H(F) \leq 2^{t+1}\right\}
$$


and choose the parameters $\alpha$ and $\beta$ as follows:

$$
\begin{aligned}
& 0<\alpha<\gamma / 4, \\
& \alpha / 2<\beta<\alpha .
\end{aligned}
$$

For every $t$ we divide $I$ into intervals $I_{s}^{t}$ of length $c 2^{t(-1-\gamma / 2+\alpha)}$ each, where $c=c(t) \in[1,2]$. Let

$$
\left[I_{s}^{t}\right]_{t}=\left\{F \in \mathcal{G}(t): \sigma(F) \cap I_{s}^{t} \neq \emptyset\right\} .
$$

If $F \in\left[I_{s}^{t}\right]_{t}$, then by Taylor's formula, (55) and (56), we get

$$
\begin{gathered}
|F(x)| \ll 2^{t(-1-\gamma+2 \alpha)}, \\
\left|F^{\prime}(x)\right| \ll 2^{t(-\gamma / 2+\alpha)}
\end{gathered}
$$

for all $x \in \widehat{I}_{s}^{t}$.

Consider the following four types of intervals:

1) $I_{s}^{t}$ is called of type $A$ if $\left|\left[I_{s}^{t}\right]_{t}\right| \leq 2^{\alpha t / 2}$.

2) $I_{s}^{t}$ is called of type $B$ if for any distinct $F_{1}, F_{2} \in\left[I_{s}^{t}\right]_{t}$,

$$
d\left(F_{1}, F_{2}\right) \leq 2^{t(-1-\gamma / 2+\beta)},
$$

where $d\left(F_{1}, F_{2}\right)=d\left(\sigma\left(F_{1}\right), \sigma\left(F_{2}\right)\right)$.

3) $I_{s}^{t}$ is called of type $C$ if there exist $F_{\mathbf{A}}, F_{\mathbf{B}}, F_{\mathbf{C}} \in\left[I_{s}^{t}\right]_{t}$ such that

$$
\left|\begin{array}{lll}
a_{0} & a_{1} & a_{2} \\
b_{0} & b_{1} & b_{2} \\
c_{0} & c_{1} & c_{2}
\end{array}\right| \neq 0
$$

with $\mathbf{A}=\left(a_{0}, a_{1}, a_{2}\right), \mathbf{B}=\left(b_{0}, b_{1}, b_{2}\right)$ and $\mathbf{C}=\left(c_{0}, c_{1}, c_{2}\right)$.

4) If $I_{s}^{t}$ is not of type A, B or C, then it is called of type $D$.

Assertion 1. The measure of those $x \in I$ which belong to infinitely many $\sigma(F)$, where $F \in\left[I_{s}^{t}\right]_{t}$ and $I_{s}^{t}$ is a type $A$ or $B$ interval, is equal to zero.

Proof. Counting the number of $F$ for type A intervals $I_{s}^{t}$ with a fixed $t$ we get

$$
\sum_{F \in \mathcal{G}(t)} \mu \sigma(F) \ll 2^{t(-1-\gamma / 2)} 2^{t(1+\gamma / 2-\alpha)} 2^{\alpha t / 2}=2^{-\alpha t / 2} .
$$

The Borel-Cantelli lemma finishes the proof in this case. Let $I_{s}^{t}$ be a type B interval. By (66) there exists an interval $\Delta_{s}^{t}$ of length $\ll 2^{t(-1-\gamma / 2+\beta)}$ such that

$$
\bigcup_{F \in\left[I_{s}^{t}\right]_{t}} \sigma(F) \subset \Delta_{s}^{t}
$$


Then counting the number of intervals $I_{s}^{t}$ we get

$$
\begin{aligned}
\sum_{t \geq 0} \sum_{s} \mu\left(\bigcup_{F \in\left[I_{s}^{t}\right]_{t}} \sigma(F)\right) & \ll \sum_{t \geq 0} 2^{t(1+\gamma / 2-\alpha)} 2^{t(-1-\gamma / 2+\beta)} \\
& \ll \sum_{t \geq 0} 2^{-(\alpha-\beta) t}<\infty .
\end{aligned}
$$

The Borel-Cantelli lemma finishes the proof.

Now if $x_{0} \in I$ belongs to infinitely many $\sigma(F)$, where $F \in\left[I_{s}^{t}\right]_{t}$ with $I_{s}^{t}$ an interval of type $\mathrm{C}$ or $\mathrm{D}$, then $x_{0}$ belongs to $\widehat{I}_{s}^{t}$ for infinitely many $t$, where $I_{s}^{t}$ is a type $\mathrm{C}$ or D interval.

AsSERTION 2. The measure of those $x \in I$ which belong to infinitely many $\widehat{I}_{s}^{t}$, where $I_{s}^{t}$ is a type $C$ interval, is equal to zero.

Proof. We consider a type $\mathrm{C}$ interval $I_{s}^{t}$. There exist $F_{\mathbf{A}}, F_{\mathbf{B}}, F_{\mathbf{C}} \in\left[I_{s}^{t}\right]_{t}$ satisfying (67). For rational integers $p_{1}, p_{2}, p_{3}$ such that $\left|p_{i}\right| \leq 2^{t / 3}(i=$ $1,2,3)$, we consider expressions of the form

$$
p_{1} a_{2}+p_{2} b_{2}+p_{3} c_{2} \text {. }
$$

Their values belong to some interval $\left[-C_{6} 2^{t+t / 3}, C_{6} 2^{t+t / 3}\right]$, where $C_{6}$ is a constant independent of $t$. The number of different expressions of the form $(68)$ is $\asymp 2^{t}$. Dirichlet's principle implies the existence of two different expressions of the form (68) with difference $\ll 2^{t / 3}$. Let $p_{10} a_{2}+p_{20} b_{2}+p_{30} c_{2}$ denote this difference. It is obvious that

$$
\left|p_{10}\right|+\left|p_{20}\right|+\left|p_{30}\right| \neq 0 \text {. }
$$

We define $R(x)=p_{10} F_{\mathbf{A}}(x)+p_{20} F_{\mathbf{B}}(x)+p_{30} F_{\mathbf{C}}(x)$. From (69) and (67) we have $R(x) \not \equiv 0$. Moreover, $R(x)=a_{0}(R)+a_{1}(R) f_{1}+a_{2}(R) f_{2}$ and

$$
\left|a_{2}(R)\right| \ll 2^{t / 3} \text {. }
$$

The estimates (64), (65) and the definition of $R$ yield

$$
\begin{aligned}
& |R(x)| \ll 2^{t(-2 / 3-\gamma+2 \alpha)}, \\
& \left|R^{\prime}(x)\right| \ll 2^{t(1 / 3-\gamma / 2+\alpha)}
\end{aligned}
$$

for all $x \in \widehat{I}_{s}^{t}$. The exponents in (71) and (72) are less than $t / 3$. Hence from (70) we obtain $H(R) \ll 2^{t / 3}$ and then from (71) we have

$$
|R(x)| \ll H(R)^{-2-(3 \gamma-6 \alpha)} .
$$

The exponent satisfies the inequality $-2-(3 \gamma-6 \alpha)<-2$. Therefore the proof is finished by Schmidt's theorem.

Consider a type D interval $I_{s}^{t}$. It has the following properties:

(a) $\left|\left[I_{s}^{t}\right]_{t}\right|>2^{\alpha t / 2}$;

(b) there exist $F_{\mathbf{A}}, F_{\mathbf{B}} \in\left[I_{s}^{t}\right]_{t}$ such that $d\left(F_{\mathbf{A}}, F_{\mathbf{B}}\right)>2^{t(-1-\gamma / 2+\beta)}$; 
(c) for any $F_{\mathbf{A}}, F_{\mathbf{B}}, F_{\mathbf{C}} \in\left[I_{s}^{t}\right]_{t}$ condition (67) does not hold.

By (c) there exists a plane with the normal $\mathbf{N}_{s}$ such that $\left(\mathbf{N}_{s}, \mathbf{A}\right)=0$ for any $F_{\mathbf{A}} \in\left[I_{s}^{t}\right]_{t}$. Let $F_{\mathbf{A}}, F_{\mathbf{B}} \in\left[I_{s}^{t}\right]_{t}$ and $d\left(F_{\mathbf{A}}, F_{\mathbf{B}}\right)>2^{t(-1-\gamma / 2+\beta)}$. Then, using (54) and (59), we obtain

$$
\left|a_{1} b_{2}-a_{2} b_{1}\right| \gg 2^{t(1-\gamma / 2+\beta)} .
$$

By definition $\mathbf{A} \times \mathbf{B}=\left(a_{1} b_{2}-a_{2} b_{1}, a_{2} b_{0}-a_{0} b_{2}, a_{0} b_{1}-a_{1} b_{0}\right)$. Then from (74) we have

$$
|\mathbf{A} \times \mathbf{B}| \gg 2^{t(1-\gamma / 2+\beta)}
$$

Moreover,

It is known that

$$
\mathbf{N}_{s}= \pm \frac{\mathbf{A} \times \mathbf{B}}{|\mathbf{A} \times \mathbf{B}|}
$$

$$
\mathbf{A} \times(\mathbf{B} \times \mathbf{C})=(\mathbf{B}, \mathbf{A}) \mathbf{C}-(\mathbf{C}, \mathbf{A}) \mathbf{B}
$$

where $\mathbf{A}, \mathbf{B}, \mathbf{C} \in \mathbb{R}^{3}$. It is obvious that $(\mathbf{F}(x), \mathbf{A})=F_{\mathbf{A}}(x)$. Then for $x \in \widehat{I}_{s}^{t}$ we find

$$
\begin{aligned}
\mathbf{F}(x) \times \mathbf{N}_{s} & = \pm \mathbf{F}(x) \times \frac{\mathbf{A} \times \mathbf{B}}{|\mathbf{A} \times \mathbf{B}|}= \pm \frac{1}{|\mathbf{A} \times \mathbf{B}|}((\mathbf{A}, \mathbf{F}(x)) \mathbf{B}-(\mathbf{B}, \mathbf{F}(x)) \mathbf{A}) \\
& = \pm \frac{1}{|\mathbf{A} \times \mathbf{B}|}\left(F_{\mathbf{A}}(x) \mathbf{B}-F_{\mathbf{B}}(x) \mathbf{A}\right) .
\end{aligned}
$$

Further, using the estimates (64), (75) and $|\mathbf{A}| \ll 2^{t},|\mathbf{B}| \ll 2^{t}$, we get

$$
\left|\mathbf{F}(x) \times \mathbf{N}_{s}\right| \ll 2^{t(-1+\gamma / 2-\beta)} 2^{t} 2^{t(-1-\gamma+2 \alpha)} .
$$

Thus we have

$$
\left|\mathbf{F}(x) \times \mathbf{N}_{s}\right| \ll 2^{t(-1-\gamma / 2+2 \alpha-\beta)}
$$

for all $x \in I_{s}^{t}$.

ASSERTION 3. The measure of those $x \in I$ which belong to infinitely many $\widehat{I}_{s}^{t}$, where $I_{s}^{t}$ is a type D interval, is equal to zero.

Proof. A type D interval $I_{s}^{t}$ is called a subtype $D_{1}$ interval if there does not exist a type D interval $I_{h}^{t}(s \neq h)$ such that

$$
2^{t(-1-\gamma / 2+3 \alpha / 2)} \leq d\left(I_{s}^{t}, I_{h}^{t}\right) \leq 2^{-1-\gamma / 2+2 \alpha} .
$$

The other type $\mathrm{D}$ intervals are subtype $D_{2}$ intervals. The number of subtype $\mathrm{D}_{1}$ intervals is $\ll 2^{t(1+\gamma / 2-3 \alpha / 2)}$. Hence

$$
\sum_{t \geq 0} \sum_{s} \mu \widehat{I}_{s}^{t} \ll \sum_{t \geq 0} 2^{\alpha t / 2}<\infty .
$$

The Borel-Cantelli lemma finishes the proof in this case. Further, let $I_{s}^{t}$ be a subtype $\mathrm{D}_{2}$ interval. There exists a type D interval $I_{h}^{t}$ satisfying (78). Let 
$\Delta_{s, h}^{t}$ denote the smallest interval containing both $I_{s}^{t}$ and $I_{h}^{t}$. From (75) we get

$$
\mu \Delta_{s, h}^{t} \ll 2^{t(-1-\gamma / 2+2 \alpha)} .
$$

If there exist $F_{\mathbf{A}}, F_{\mathbf{B}}, F_{\mathbf{C}} \in\left[\Delta_{s, h}^{t}\right]_{t}$ such that (67) holds then we obtain a bigger type $\mathrm{C}$ interval. The choice of $\alpha$ and (79) yield the following fact: the set of those $x \in I$ which belong to infinitely many such intervals has measure zero as in the proof of Assertion 2.

In the last case the normals coincide: $\mathbf{N}=\mathbf{N}_{s}=\mathbf{N}_{h}$. Using (13) and (77) for $x \in I_{s}^{t}$ and $y \in I_{h}^{t}$ we find

$$
\begin{aligned}
|x-y| & \asymp\left|f_{1}(x)-f_{1}(y)\right| \leq|\mathbf{F}(x) \times \mathbf{F}(y)| \\
& \ll|\mathbf{F}(x) \times \mathbf{N}|+|\mathbf{F}(y) \times \mathbf{N}| \ll 2^{t(-1-\gamma / 2+2 \alpha-\beta)} .
\end{aligned}
$$

The last inequality and (78) give

$$
2^{t(-1-\gamma / 2+3 \alpha / 2)} \ll|x-y| \ll 2^{t(-1-\gamma / 2+2 \alpha-\beta)} .
$$

The choice of $\beta$ in (62) shows that $(-1-\gamma / 2+3 \alpha / 2)>(-1-\gamma / 2+2 \alpha-\beta)$. Hence inequality (80) is contradictory for $t$ large. Assertion 3 is proved. Thus Proposition 4 is proved.

7. Completion the proof of the Theorem. Let $\lambda=\min \left(\varepsilon / 8, \varepsilon^{2} / 16\right)$. Applying Propositions $1-4$ at most $[8 / \lambda]+1$ times we get

$$
\mu \Psi_{3}(\varepsilon) \leq \mu \Psi_{3}\left(\varepsilon_{1}\right)
$$

where $\varepsilon_{1}>8$. By Remark 4 the proof of the Theorem is complete.

The research described in this paper was made possible in part by Grant No. MWP000 from the International Science Foundation and was partially supported by the International Soros Science Education Program.

\section{References}

[1] A. Piartly, Diophantine approximations on submanifolds of Euclidean space, Funktsional. Anal. i Prilozhen. 3 (4) (1969), 59-62 (in Russian).

[2] W. M. Schmidt, Metrische Sätze über simultane Approximation abhängiger Grössen, Monatsh. Math. 63 (1964), 154-166.

Institute of Mathematics

Academy of Sciences of Belarus

220072 Minsk, Belarus

E-mail: imanb\%imanb.belpak.minsk.by@demos.su 